\title{
Indecomposable Continua and Misiurewicz Points in Exponential Dynamics
}

\author{
Robert L. Devaney* \\ Mathematics Department \\ Boston University \\ Boston, MA 02215, USA
}

\author{
Xavier Jarque J $^{\dagger}$ \\ Dep. Mat. Aplicada i Anàlisi \\ Universitat de Barcelona \\ Gran Via 585 \\ 08007 Barcelona, Spain
}

\author{
Mónica Moreno Rocha \\ Mathematics Department \\ Tufts University \\ Medford, MA 02155, USA
}

January 19, 2005

\section{Introduction}

In this paper we describe several new types of invariant sets that appear in the Julia sets of the complex exponential functions $E_{\lambda}(z)=\lambda e^{z}$ where $\lambda \in \mathbb{C}$. These invariant sets consist of points that share the same itinerary under iteration of $E_{\lambda}$. Since these exponential functions are $2 \pi i$ periodic, there are several "natural" ways (described below) to decompose the plane into countably many strips of vertical height $2 \pi$ which are then indexed in the natural way by the integers according to the increasing imaginary parts of the strips. The itinerary of a point is then the sequence of integers that describes how the orbit of that point passes through these various strips. Thus we investigate the sets of points whose orbits make the transit through these strips in the same fashion.

For complex analytic maps, the Julia set consists of all points at which the family of iterates of the map fails to form a normal family in the sense of Montel. Equivalently, the Julia set may be described as either the closure of the set of repelling periodic points or else as the set of points on which the map behaves chaotically. For $E_{\lambda}$, the Julia set is also the closure of the set of points whose orbits tend to $\infty$ [17]. We denote the Julia set of the exponential map by $J\left(E_{\lambda}\right)$. It is well known that, if $J\left(E_{\lambda}\right)$ contains an open set, then in fact the Julia set must be the entire plane. Otherwise, $J\left(E_{\lambda}\right)$ is a nowhere dense subset of the plane.

\footnotetext{
*Partially supported by NSF Grant 02-05779.

${ }^{\dagger}$ Partial support from the Spanish Ministry of education and Science and FEDER through the grants BFM200201344 and SEC2003-00306 and from the Catalan Government through the grant SGR99-00349 is gratefully acknowledge.

${ }^{\ddagger}$ Supported by FRAC Grant, 2003, Tufts University.
} 
In the latter case, it is known [5], [12] that the set of points that share the same itinerary must lie on hairs in the plane. A hair is a simple, continuous curve extending to $\infty$ in the right half plane and having a distinguished endpoint. All non-endpoints of these curves are points whose orbits tend to $\infty$ in the right half plane. Hence all points with bounded orbits must lie at the endpoints of these curves. In particular, since the repelling periodic points are dense in the Julia set, it follows that the endpoints of these curves must be dense in $J\left(E_{\lambda}\right)$. In the case where $E_{\lambda}$ admits an attracting fixed point, it is known that the Julia set is a Cantor bouquet [12], [18], an extremely rich type of topological space, [1]. When the Julia set is nowhere dense but $E_{\lambda}$ does not have an attracting fixed point, the Julia set is still a type of Cantor bouquet, but now distinct hairs may share the same endpoint. These structures have been described in [3].

The situation is quite different when the Julia set of $E_{\lambda}$ is the entire plane. This may occur in a number of ways. For example, if the orbit of the asymptotic value 0 tends to $\infty$, then we must have $J\left(E_{\lambda}\right)=\mathbb{C}$. This situation occurs when 0 lies on a (non-endpoint of a) hair. In this case, it is known [7], [11], [21] that the sets of points corresponding to certain itineraries is no longer a hair but rather an indecomposable continuum. An indecomposable continuum is another example of a complicated topological space which is defined as a continuum that cannot be written as the union of two proper subcontinua. These indecomposable continua arise when a hair in the Julia set becomes so entangled that it accumulates everywhere on itself.

There is another way that $J\left(E_{\lambda}\right)$ may be the entire plane. If the orbit of 0 eventually lands on a repelling periodic orbit, then it is known that $J\left(E_{\lambda}\right)=\mathbb{C}$. Such a $\lambda$-value is called a Misiurewicz parameter. In this case it is known that many of the hairs share the same endpoint (see [10]).

It is here, when $\lambda$ is preperiodic, that we find several new types of sets that share the same itinerary. In [22] D. Schliecher pointed out that Theorem 3.3 (and consequently Theorem 3.6) of [10] cannot be applied to certain dynamic sequences for which an indecomposable continua would arise. The subject of this paper is to provide the construction and to characterize the sets that give rise for such sequences. Precisely, one new type is an indecomposable continuum that differs from the continuum described above in that we have a pair of hairs that accumulate upon each other, rather than a single hair having this property. The second type consists of an indecomposable continuum together with a completely separate hair that accumulates on this continuum. We note that this special kind of indecomposable continua with more than one curve involved is not new in dynamics since such sets can be constructed by using homoclinic and heteroclinic connections of hyperbolic saddles for diffeomorphisms of the plane.

Rather than deal with the general Misiurewicz case, we shall deal in this paper only with the very special case where $\lambda=2 \pi i$. In this case, the orbit of $z=0$ lands at $z=2 \pi i$, which is a repelling fixed point for the map.

\section{Preliminaries.}

In this section we introduce the basic definitions and notation that we shall use in following sections. We also summarize some well known facts concerning the construction of "hairs" in the dynamical 
plane. The dynamics of the exponential family are well understood if $\lambda$ is real. See [5], [11], and [12]. There are no Misiurewicz parameters in the case of real $\lambda$-values; hence we shall restrict our attention in this paper to the case where $\lambda \in \mathbb{C} \backslash \mathbb{R}$.

For such a $\lambda$-value, the horizontal lines given by $y=x+i(2 k-1) \pi-i \arg \lambda, x \in \mathbb{R}$, are mapped to the negative real axis by $E_{\lambda}$. These lines partition the plane into a collection of open horizontal strips called fundamental domains which we denote by $R_{k}, k \in \mathbb{Z}$. Since $\lambda$ is not real, the asymptotic value $z=0$ always belongs to one of these strips which we choose to call $R_{0}$. The $R_{k}$ are then indexed in the natural way with increasing imaginary parts.

Using the above partition, we say that the sequence of integers $s=\left(s_{0}, s_{1}, \ldots\right)$ is the itinerary of the point $z \in \mathbb{C}$ if $E_{\lambda}^{j}(z) \in R_{s_{j}}$ for each $j$. In the sequel we use the words itinerary and sequence interchangeably. For a fixed positive integer $M$ we define the set $\Sigma_{M}$ to be

$$
\Sigma_{M}=\left\{s=\left(s_{0}, s_{1}, \ldots\right)|| s_{j} \mid \leq M \text { for each } j\right\}
$$

and denote by $I(s)$ the set of points sharing a given itinerary $s \in \Sigma_{M}$. Among all sequences in $\Sigma_{M}$, the so-called regular sequences, i.e., those for which $s_{j} \neq 0$ for all $j$ will play an important role.

Fix $M \geq 1$. For $x>0$, define the half strip

$$
H_{x}=\{z \mid \operatorname{Re} z \geq x,-(2 M+1) \pi-\arg \lambda<\operatorname{Im} z \leq(2 M+1) \pi-\arg \lambda\} .
$$

We say that the orbit of $z$ tends directly to $\infty$ in $H_{x}$ if the entire orbit of $z$ lies in $H_{x}$ and $\operatorname{Re} E_{\lambda}^{n+1}(z)>\operatorname{Re} E_{\lambda}^{n}(z)$ for all $n$. Let $\omega_{s}(x)$ denote the set of points in $H_{x}$ whose orbits tend directly to $\infty$ with itinerary $s \in \Sigma_{M}$. It follows from [5] that there exists $\zeta \in \mathbb{R}^{+}$such that $\omega_{s}=\omega_{s}(\zeta)$ is a continuous curve of the form $\left(t, h_{s}(t)\right)$ with $\zeta \leq t<\infty$. The value $\zeta$ depends only on $M$, not the particular sequence $s \in \Sigma_{M}$. We call $\omega_{s}$ the tail of $I(s)$. Note that, by definition, if $z \in \omega_{s}$, then $E_{\lambda}^{n}(z) \in H_{\zeta}$ for all $n \geq 0$.

By choosing $\zeta$ larger if necessary, we may assume that $E_{\lambda}$ maps the vertical line $\operatorname{Re} z=\zeta$ to a circle that crosses both horizontal boundaries of $H_{\zeta}$ at points with real parts strictly larger than $\zeta$. That is, the circle of radius $|\lambda| e^{\zeta}$ intersects $H_{\zeta}$ to the right of the line $\operatorname{Re} z=\zeta$. Hence we define the fundamental domain for the tails as

$$
F_{\zeta}=\left\{z \in H_{\zeta}|| z|<| \lambda \mid e^{\zeta}\right\}
$$

The portion of $\omega=\omega_{s}(\zeta)$ contained in $F_{\zeta}$, denoted here by $\alpha_{s}=\alpha_{s}(\zeta)$, is called the base of the tail. See Figure 1. It is well known (see [5]) that $\left\{\alpha_{s} \mid s \in \Sigma_{M}\right\}$ is homeomorphic to the product of a Cantor set and the interval $[0,1)$.

We now fix $s$. To determine the set $I(s)$, we pull back the curve $\omega_{s}$ to produce a longer curve (that will not in general be a graph of the form $\left(t, h_{s}(t)\right)$ as is $\left.\omega_{s}\right)$, each point of which will have itinerary $s \in \Sigma_{M}$. To accomplish this, we use the shift map $\sigma: \Sigma_{M} \rightarrow \Sigma_{M}$ defined as $\sigma\left(s_{0} s_{1} s_{2} \ldots\right)=\left(s_{1} s_{2} s_{3} \ldots\right)$. Hence $\omega_{\sigma(s)}$ is a tail that properly contains the curve $E_{\lambda}\left(\omega_{s}\right)$ since $E_{\lambda}\left(\omega_{s}\right)$ misses the base $\alpha_{\sigma(s)}$. Consider $L_{s_{0}}\left(\omega_{\sigma(s)}\right)$, where $L_{s_{0}}$ is the branch of the logarithm taking 


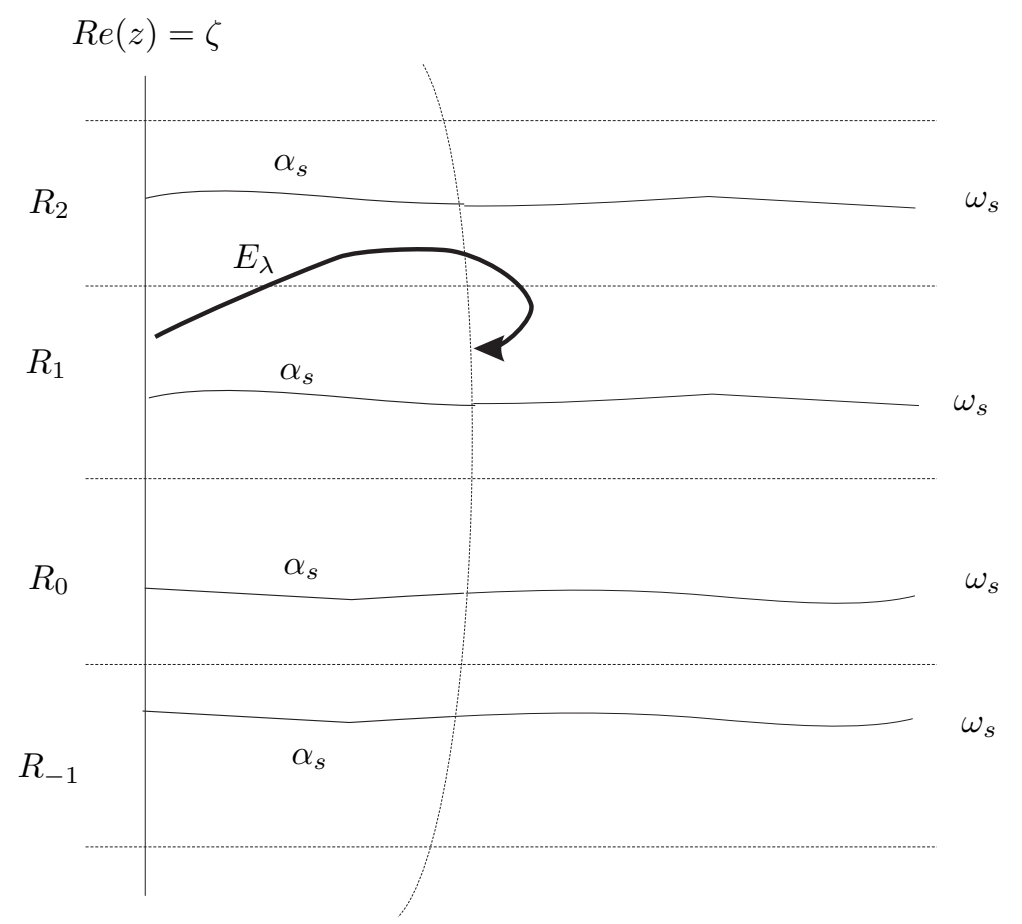

Figure 1: The set $H_{\zeta}$, the tail $\omega_{s}$ and the base $\alpha_{s}$ for some sequence $s \in \Sigma_{M}$.

values in $R_{s_{0}}$. This is a continuous curve that lies in $R_{s_{0}}$ and extends $\omega_{s}$. Clearly, any $z \in L_{s_{0}}\left(\omega_{\sigma(s)}\right)$ has itinerary $s$.

Inductively, consider

$$
L_{s_{0}} \circ \ldots \circ L_{s_{n-1}}\left(\omega_{\sigma^{n}(s)}\right) .
$$

This backward induction is well defined unless this curve meets $z=0$; however, as long as $s$ is a regular sequence, this backward process is far from $z=0$. Hence this curve, by construction, contains points with itinerary $s$, and each such curve extends its predecessor. Let

$$
\gamma(s)=\bigcup_{n=0}^{\infty} L_{s_{0}} \circ \ldots \circ L_{s_{n-1}}\left(\omega_{\sigma^{n}(s)}\right) .
$$

We call $\gamma(s)$ the hair associated to $s$. Of course $\gamma(s) \subset I(s)$. The following result for regular sequences is proved in [5].

Theorem 2.1. Let $s$ be a regular sequence. Then set $I(s)$ is the union of $\gamma(s)$ (the hair) and a single point $z_{s}$ (the end point of the hair) whose orbit is bounded.

The above theorem fully characterizes the set $I(s)$ for any $\lambda$ in $\mathbb{C}$ and any regular sequence $s$, and shows that the pullbacks of the curve $\omega_{s}$ described above lands on a unique point with bounded orbit and itinerary $s$. 
For most non-regular sequences, this situation is not true. On one hand, we may have, by choosing convenient "new" fundamental domains, more than one tail following the sequence $s$ and tending directly to infinity. One the other hand, the pullback process described above may not land at a particular end point but may become quite entangled and topologically very rich. The aim of the paper is to show that this occurs in the case of Misiurewicz parameters, and precisely for $\lambda=2 \pi i$.

\section{The dynamical plane for $\lambda=2 \pi i$}

For the remainder of this paper, we restrict attention to the case where $\lambda=2 \pi i$. Note that $E_{2 \pi i}(0)=2 \pi i$, which is a repelling fixed point for $E_{2 \pi i}$. Hence this is a Misiurewicz parameter value. From now on we denote $E_{2 \pi i}$ by $E$.

The point $2 \pi i$ is a repelling fixed point with regular sequence $s=(111 \ldots)$ with respect to the fundamental domains $R_{k}, k \in \mathbb{Z}$. Consequently, from Theorem 2.1, there is a unique hair, denoted by $\gamma_{2 \pi i}$, corresponding to this sequence which lands at the endpoint $z=2 \pi i$.

Since 0 maps onto $2 \pi i$ after one iteration of $E$, there is a preimage of $\gamma_{2 \pi i}$ which is a hair attached to 0 . We denote this curve by $\gamma_{0}$. The preimage of $\gamma_{0}$ consists of infinitely many disjoint curves, each of which extends from left to right across the entire plane. We denote these curves by $t_{j}, j \in \mathbf{Z}$. We again index the $t_{j}$ so that $j$ increases with increasing imaginary part (note that $t_{j+1}=t_{j}+2 \pi i$ for each $j$ ). In particular, we choose $t_{0}$ so that 0 lies in the region bounded by $t_{0}$ and $t_{1}$. Therefore $2 \pi i$ lies in the strip bounded by $t_{1}$ and $t_{2}$.

We remark that $t_{0}$ is asymptotic to the horizontal line $y=-\frac{\pi}{2}$ in the far right hand plane. Consequently, $t_{j}$ is asymptotic to $y=-\frac{\pi}{2}+2 \pi j$ for each $j$. We also remark that, since $E^{\prime}(2 \pi i)=$ $2 \pi i$, it can be shown that $\gamma_{2 \pi i}$ spirals around $2 \pi i$ in the clockwise direction. Therefore, the $t_{j}$ are curves whose imaginary parts decrease in the far left half plane as depicted in Figure 2.

The curves $t_{j}$ provide us with a new set of fundamental domains in which we can define, as before, itineraries for $E$. We denote by $T_{j}, j \in \mathbf{Z}$, the open strip bounded above by $t_{j+1}$ and below by $t_{j}$. Note that $E$ maps $T_{j}$ onto $\mathbb{C}-\left\{\gamma_{0}\right\}$ in one-to-one fashion. The itinerary of $z$ in these new fundamental domains given by the $T_{j}$ 's is defined as in the above case of $R_{j}$ 's. In particular, the points $z$ lying on the curves $t_{j}$ have itinerary $(j-1) 011 \ldots$ The $T_{j}$ fundamental regions are more natural (and useful) for coding itineraries than the previous $R_{k}$ regions since the boundaries of the strips are now dynamically defined. In [10] it is shown that the topological structure of the set of all points sharing a specific itinerary with respect to the $T_{j}$ partition, depends only on the symbols of the itinerary.

To do so, we split the symbols 0 and 1 to $0^{1}, 0^{2}$ and $1^{1}, 1^{2}$, respectively. Precisely, the symbols $0^{1}$ and $0^{2}$ specify when the orbit of a point passes through $T_{0}$ below or above $\gamma_{0}$, respectively. Similarly, the symbols $1^{1}$ or $1^{2}$ specify that, far to the right, a point passes through $T_{1}$ below or above $\gamma_{2 \pi i}$, respectively. As a consequence, far to the right, we consider the augmented partition $\bar{T}_{j}, j \in\left\{-M, \ldots,-1,0^{1}, 0^{2}, 1^{1}, 1^{2}, 2, \ldots, M\right\}$.

Further, we say that $s=\left(s_{0}, s_{1}, \ldots\right)$ is an $A$-sequence (respectively $B$-sequence) if it con- 


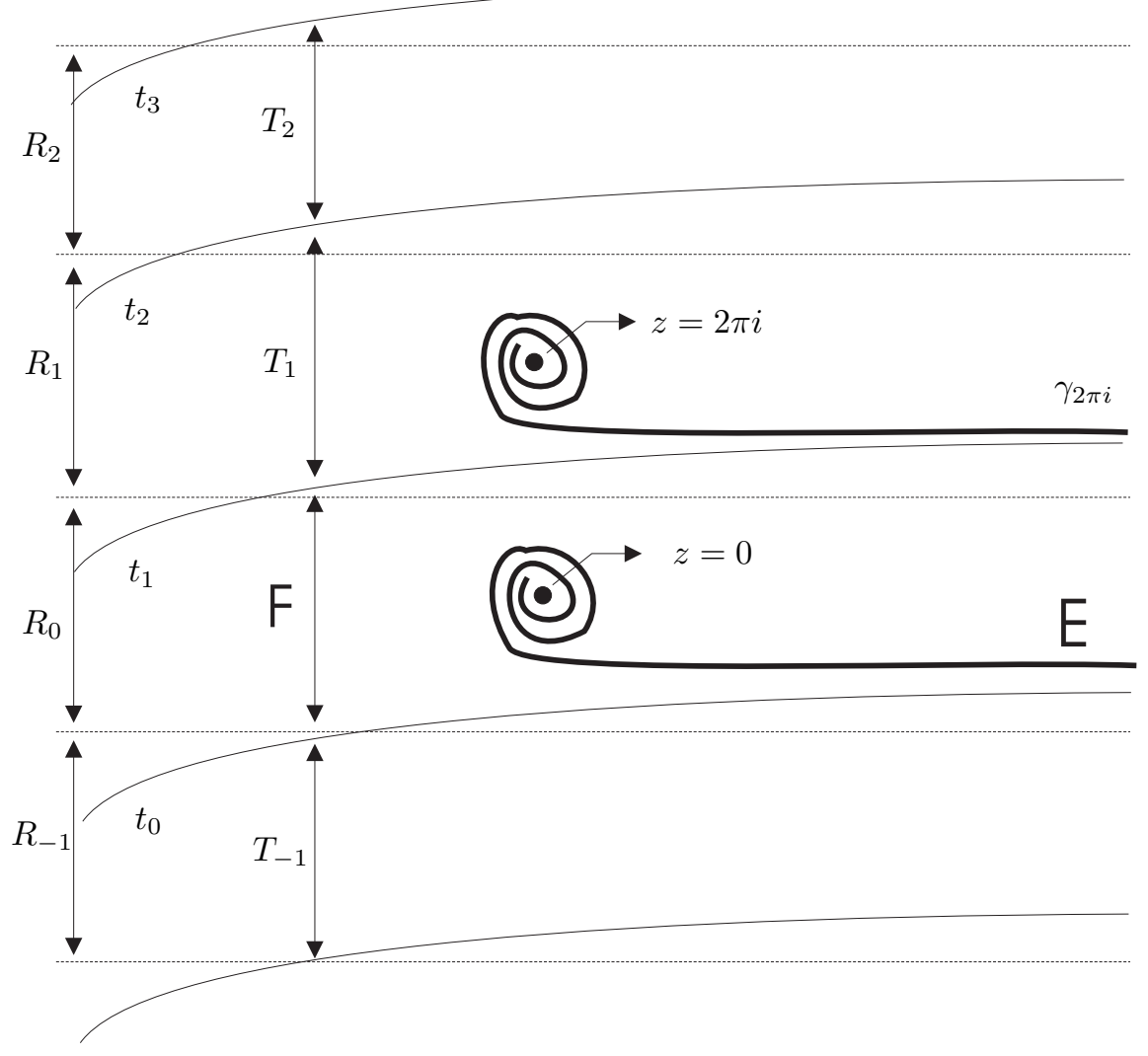

Figure 2: The two families of fundamental domains $R_{j}$ and $T_{j}$ and the hairs $\gamma_{0}$ and $\gamma_{2 \pi i}$. Under the action of $E$, the boundaries of the domains $T_{j}$ are mapped to $\gamma_{0}, \gamma_{0}$ is mapped to $\gamma_{2 \pi i}$ and $\gamma_{2 \pi i}$ is mapped to itself.

tains infinitely many (respectively, finitely many) symbols $s_{j} \in\{-M, \ldots, 0,1, \ldots M\}$, different from 0 and 1. Similarly, we say that $\bar{s}=\left(s_{0}, s_{1}, \ldots\right)$ is an $\bar{A}$-sequence (respectively $\bar{B}-$ sequence) if it contains infinitely many (respectively, finitely many) augmented symbols $s_{j} \in$ $\left\{-M, \ldots, 0^{1}, 0^{2}, 1^{1}, 1^{2}, \ldots M\right\}$, different from $0^{1}, 0^{2}, 1^{1}$ and $1^{2}$. Note that, by taking preimages, we may reduce the study of $B-\left(\right.$ and $\bar{B}-$ ) sequences to sequences with (only) $\{0,1\}$ (and $\left\{0^{1}, 0^{2}, 1^{1}, 1^{2}\right\}$ ) symbols, respectively. In what follows, we will only consider this case.

Finally, we use the notation $s=\left(s_{0}, s_{1}, \ldots\right) \in \Sigma_{M}$ when referring to either an $A$ - or $B$ sequence without the augmented symbols, and $\bar{s}=\left(\bar{s}_{0}, \bar{s}_{1}, \ldots\right) \in \bar{\Sigma}_{M}$ when referring to either an $\bar{A}-$ or $\bar{B}$-sequence with the augmented symbols.

Remark 3.1. There is a major difference between the $A$ - and $B$-sequences and $\bar{A}-$ and $\bar{B}$ sequences. Indeed, not every augmented sequence is "admissible", that is, for some $\bar{s}$ there are no points $z \in \mathbb{C}$ whose orbit tends directly to $\infty$ following such sequence. The transition matrix for 
$\bar{B}$-sequences is given by

$$
L=\left(\begin{array}{llll}
0 & 1 & 1 & 0 \\
1 & 0 & 0 & 1 \\
0 & 1 & 1 & 0 \\
1 & 0 & 0 & 1
\end{array}\right)
$$

where the rows and columns of $L$ correspond in order to $0^{1}, 0^{2}, 1^{1}$ and $1^{2}$, so $l_{i j}=1$ if and only if $i \rightarrow j$. For example, $l_{0^{1} 0^{1}}=0$ since $0^{1} \nrightarrow 0^{1}$ but $l_{0^{1} 0^{2}}=1$ since $0^{1} \rightarrow 0^{2}$. The (non) admissibility of $\bar{A}$ - and $\bar{B}$-sequences make sense when looking for points in $\mathbb{C}$ whose orbit tends directly to infinity in the asymptotic direction. In subsequent sections we will see that there are points following "non admissible" sequences but not tending directly to infinity.

Remark 3.2. A sequence $s$ and its augemtation $\bar{s}$ are related as follows. Let $\pi: \bar{\Sigma}_{M} \rightarrow \Sigma_{M}$ be the projection map given by simply erasing the superscripts. If $s$ is an A-sequence, then there exists a unique admissible $\bar{A}$-sequence, $\bar{s}$, such that $\pi(\bar{s})=s$, while if $s$ is a $B$-sequence, then there exist exactly two admissible $\bar{B}$-sequences, $\bar{s}_{1}$ and $\bar{s}_{2}$ such that $\pi\left(\bar{s}_{i}\right)=s, i=1,2$. For instance, the two admissible $\bar{B}$-sequences corresponding to the $B$-sequence $s=(1,0,1,0, \ldots)$ are $\bar{s}_{1}=\left(1^{1}, 0^{2}, 1^{2}, 0^{1}, \ldots\right)$ and $\bar{s}_{2}=\left(1^{2}, 0^{1}, 1^{1}, 0^{2}, \ldots\right)$, while the unique admissible $\bar{A}$-sequence corresponding to the $A$-sequence $s=(0,1,2,0,1,2, \ldots)$ is $\bar{s}=\left(0^{2}, 1^{2}, 2,0^{2}, 1^{2}, 2, \ldots\right)$.

\subsection{Hairs in $T_{j}$}

We redefine as in the previous section the set $H_{x}$ with respect the new partition $T_{j}$ where the top and bottom horizontal boundaries are now given by $t_{M+1}$ and $t_{-(M+1)}$. By choosing $\zeta$ large enough, $E$ maps the vertical line $\operatorname{Re} z=\zeta$ to a circle that crosses these boundaries at points with real parts strictly larger than $\zeta$.

In [10] it is shown that the set of points in $H_{\zeta}$ whose orbit tends directly to $\infty$ with itinerary $s$ has a different structure depending upon the sequence $s$. More precisely, we have

Lemma 3.1. Choose $\zeta>0$ large enough.

(a) If $s$ is an $A$-sequence, then the set of points, $\omega_{s}$, lying in $H_{\zeta}$ whose orbit tends directly to $\infty$ (inside $H_{\zeta}$ ) with itinerary $s$ is a continuous curve extending to infinity.

(b) If $s$ is a $B$-sequence ( $\bar{s}_{1}$ and $\bar{s}_{2}$ are the two corresponding $\bar{B}$-sequences), then the set of points in $H_{\zeta}$ whose orbits tend directly to $\infty$ (inside $H_{\zeta}$ ) with itinerary $s$ is the union of two disjoint continuous curves extending to infinity, denoted by $\omega_{\bar{s}_{1}}$ and $\omega_{\bar{s}_{2}}$ and following, respectively, the $\bar{B}$-sequences $\bar{s}_{1}$ and $\bar{s}_{2}$.

In particular, if s starts with 1 (respectively 0 ) then $\omega_{\bar{s}_{1}}$ lies below $\gamma_{2 \pi i}$ (respectively $\gamma_{0}$ ) and $\omega_{\bar{s}_{2}}$ lies above of $\gamma_{2 \pi i}$ (respectively $\gamma_{0}$ ).

As in Section 2, the $\omega$-curves are called tails and the set $F_{\zeta}=\left\{z \in H_{\zeta}|| z \mid<2 \pi e^{\zeta}\right\}$ is the fundamental domain of the tails. Finally, the portions of the $\omega$-curves lying in $F_{\zeta}$ are called bases 
of the tails. We let $\alpha_{s}$ denote the base of the tail $\omega_{s}$ when $s$ is an $A$-sequence, and we let $\alpha_{\bar{s}_{1}}$ and $\alpha_{\bar{s}_{2}}$ denote the bases of the tails $\omega_{\bar{s}_{1}}$ and $\omega_{\bar{s}_{2}}$ when $s$ is a $B$-sequence.

By definition, for a fixed $s$, the tail $\omega_{s}$ for $A$-sequences and the tails $\omega_{\bar{s}_{1}}$ and $\omega_{\bar{s}_{2}}$ for $B$-sequences belong to $I(s)$. To characterize the entire set $I(s)$, we need to pull these tails backward. The fact that the orbit of the asymptotic value $z=0$ lands in one iteration at $z=2 \pi i$ ensures that this backward iteration process is well defined for any $s$ that does not end in all 1's.

From the discussion above, it seems natural that this pullback procedure will produce different topological structures depending upon whether $s$ is an $A$ - or a $B$-sequence. Indeed, as in Section 2 , if $s$ is an $A$-sequence, we denote by $\gamma_{s}$ the hair associated to $s$ (that is, the limit of the backward iteration process applied to $\omega_{s}$ ), and, if $s$ is a $B$-sequence, we denote by $\gamma_{\bar{s}_{1}}$ and $\gamma_{\bar{s}_{2}}$ the hairs associated to $s$ (that is, the limit of the backward iteration processes applied to $\omega_{\bar{s}_{1}}$ and $\omega_{\bar{s}_{2}}$ ).

The following result characterizes the set $I(s)$ for a large collection of $A$ - and $B$-sequences (see $[10])$.

Proposition 3.2. Let $s=\left(s_{0}, s_{1} \ldots\right)$ be an $A$-or $B$-sequence for which there exists $K \in \mathbb{N}$ such that $s$ contains no string of consecutive 1's whose length is greater than $K$. Then

(a) If $s$ is an $A$-sequence, then the set $I(s)$ is a hair, i.e., the union of the continuous curve $\gamma(s)$ and a single point $z_{s}$ (the endpoint) whose orbit is bounded and has itinerary $s$.

(b) If $s$ is a $B$-sequence then the set $I(s)$ is the union of two disjoint continuous curves $\gamma_{\bar{s}_{1}}$ and $\gamma_{\bar{s}_{2}}$, with $\pi\left(\bar{s}_{i}\right)=s, i=1,2$. Each of these curves meet at a common endpoint $z_{s}$ whose orbit is bounded with itinerary $s$.

From the above result we conclude that, in order to complete the characterization of the sets $I(s)$, we must consider sequences with strings of 1's with arbitrarily (but finite) large length. As a preliminary observation we show that, with a single exception, the set $I(s)$ is always bounded to the left.

Lemma 3.3. Suppose that $s \in \Sigma_{M}$ and $s \neq(k, 0,1,1,1, \ldots)$ for some $k$. Then $I(s)$ lies in $\operatorname{Re}(z)>x$ for some $x>-\infty$.

Proof. Assume that $s=\left(k, 0,1, \ldots, 1, s_{l}, \ldots\right)$, where $s_{l} \neq 1$ denotes the $l$ th term of the sequence. The proof now follows by noting that points arbitrarily far to the left have sequences with an arbitrarily long string of 1 's after the 0 entry.

\section{Indecomposable continua for $\lambda=2 \pi i$}

Our main goal in this section is to show that if the lengths of the strings of 1's in a given sequence grows exponentially fast, then the set $I(s)$ is no longer a hair but rather contains an indecomposable continuum. The following theorem due to Curry [6] gives a useful criterion for the existence of an indecomposable continuum

Theorem 4.1. Suppose that $X$ is a one-dimensional nonseparating plane continuum which is the closure of a ray that limits upon itself. Then $X$ is indecomposable. 


\subsection{Targets in $H_{\zeta}$}

We now set up targets around the $n^{\text {th }}$ images of the bases $\alpha_{s}\left(\alpha_{\bar{s}_{1}}\right.$ and $\alpha_{\bar{s}_{2}}$ if $s$ is a $B$-sequence) of each tail $\omega_{s}\left(\omega_{\bar{s}_{1}}\right.$ and $\omega_{\bar{s}_{2}}$ if $s$ is a $B$-sequence). The construction is similar to the one in [11]. Let

$$
V(\xi, \eta)=\left\{z \in H_{\zeta} \mid \xi-1 \leq \operatorname{Re} z \leq \eta+1\right\} .
$$

By definition $V(\xi, \eta)$ is a "rectangle" bounded above and below by segments of $t_{M+1}$ and $t_{-M-1}$, respectively.

Proposition 4.2. Let $\zeta$ be large enough. The following statements hold.

(a) Given any $n \in \mathbb{Z}^{+}$, there exist $\xi_{n}, \eta_{n} \in \mathbb{R}^{+}$such that the $n^{\text {th }}$ iterate of all of the bases of the tails belongs to the interior of $V\left(\xi_{n}, \eta_{n}\right)$, called the $n^{\text {th }}$ target for $E$.

(b) Let $l \geq 0$. Then, for any $n \geq 0$,

$$
E\left(V\left(\xi_{n}, \eta_{n+l}\right)\right) \supset V\left(\xi_{n+1}, \eta_{n+1+l}\right) .
$$

The following proof is quite similar to the proof of Proposition 3.1 and Proposition 3.2 in [11], although, in our case, we must substitute the map $z \rightarrow e^{z}$ by $E_{2 \pi i}$. Also, note that when considering $B$-sequences, $\alpha_{s}$ is substituted by $\alpha_{\bar{s}_{1}}$ and $\alpha_{\bar{s}_{2}}$. We provide the proof of the above proposition for completeness.

Proof. First we prove (a). Since the fundamental domain for the tails $F_{\zeta}$ is contained inside $\left\{z|| z \mid=2 \pi e^{\zeta}\right\}$, we let $\xi_{0}=\zeta$ and $\eta_{0}=2 \pi e^{\zeta}$. Then $\alpha_{s} \subset V\left(\xi_{0}, \eta_{0}\right)$, for $A$-sequences, and also $\left\{\alpha_{\bar{s}_{1}}, \alpha_{\bar{s}_{2}}\right\} \subset V\left(\xi_{0}, \eta_{0}\right)$, for $B$-sequences. We also have $\left|E^{n}(z)\right| \leq E^{n+1}(\zeta)$ for each $z$ in any base of the tails. So we set $\eta_{n}=E^{n+1}(\zeta)$. Since the closure of the union of the $\alpha_{s}$ for all $s \in \Sigma_{M}$ is compact (here we also include the bases whose itinerary ends in all ones), and all orbits of points in this union move to the right under $E^{j}$, it follows that there exists a maximal $\xi_{n}$ such that Re $E^{n}(z) \geq \xi_{n}$ for each $z$ in the union of the bases of the tails.

Therefore we choose $\xi_{n}$ to be the maximal value for which the $n^{\text {th }}$ iterate of all the bases of the tails lies to the right of $\operatorname{Re} z=\xi_{n}$.

Next we prove (b). We consider the case $l=0$ (the case $l>0$ follows similarly). We choose $\zeta$ large enough so that the image of the vertical line $\operatorname{Re} z=\zeta$ meets the strip $H_{\zeta}$ in an arc whose real part is never less than $e^{\zeta}-1$. Because of this, if $w$ and $E(w)$ lie in $H_{\zeta}$, then we have $\operatorname{Re} E(w) \geq|E(w)|-1$. This follows since the image of this line is an "even more vertical" arc in $H_{\zeta}$.

Take $V\left(\xi_{n}, \eta_{n}\right)$ to be the region bounded on the left by $\xi_{n}-1$ and to the right by $\eta_{n}+1$. From the above discussion, $\xi_{n+1}$ is bigger than $2 \pi e^{\xi_{n}}-1$. Hence the left hand side boundary of $V\left(\xi_{n+1}, \eta_{n+1}\right)$ is bigger than $2 \pi e^{\xi_{n}}-2$. In contrast, $e^{\xi_{n}-1}=2 \pi e^{-1} e^{\xi_{n}}$, so $2 \pi e^{-1} e^{\xi_{n}}<2 \pi e^{\xi_{n}}-2$, as desired.

Turning our attention to the right hand side inequality, notice that $\eta_{n+1}=2 \pi e^{\eta_{n}}$. In contrast the exponential of the right hand side boundary of $V\left(\xi_{n+1}, \eta_{n+1}\right)$ has real part much bigger than $2 \pi e e^{\eta_{n}}-1>2 \pi e^{\eta_{n}}$, as desired. 
Let $s$ to be an $A$-sequence. We next enlarge the base $\alpha_{s}$ inductively to obtain an increasing sequence of curves $\alpha_{s, n}$ that satisfy the following three conditions:

(i) $\alpha_{s, 0}=\alpha_{s}$ (the base),

(ii) $\alpha_{s, n+1} \supset \alpha_{s, n}$, and

(iii) $\bigcup_{n} \alpha_{s, n}=\omega_{s}$.

To construct $\alpha_{s, 1}$, consider the bases $\alpha_{\sigma^{-1}(s)}$. This is a finite collection of bases, one in each $T_{j}, j \in\{-M, \ldots, M\}$. The set $E\left(\alpha_{\sigma^{-1}(s)}\right)$ maps into an arc of $\omega_{s}$ and to the right of $\alpha_{s}$. Let

$$
\alpha_{s, 1}=\alpha_{s, 0} \cup E\left(\alpha_{\sigma^{-1}(s)}\right),
$$

so this is a subset of $\omega_{s}$ that extends $\alpha_{s}$ to the right. It we write

$$
\alpha_{s, n}=\alpha_{s, n-1} \cup E^{n}\left(\alpha_{\sigma^{-n}(s)}\right),
$$

it is easy to check that the $\alpha_{s, n}$ have the above three properties. We call the $\alpha_{s, n}$ the $n$-initial portions of the tail.

Similarly, we expand the bases $\alpha_{\bar{s}_{1}}$ and $\alpha_{\bar{s}_{2}}$ when $s$ is a $B$-sequence, $\pi\left(\bar{s}_{i}\right)=s, i=1,2$. In this case, the above inductive procedure allows to define $\alpha_{\bar{s}_{1}, n}$ and $\alpha_{\bar{s}_{2}, n}$, respectively. Note that, for example, $\sigma^{-1}\left(1^{1}, 0^{2}, 1^{2}, 0^{1}, \ldots\right)$ consists of the two $\bar{B}$-sequences $\left(0^{1}, 1^{1}, 0^{2}, 1^{2}, 0^{1}, \ldots\right)$ and $\left(1^{1}, 1^{1}, 0^{2}, 1^{2}, 0^{1}, \ldots\right)$. Similarly, $\sigma^{-1}\left(1^{2}, 0^{1}, 1^{1}, 0^{2}, \ldots\right)$ consists of the two augmented sequences given by $\left(0^{2}, 1^{2}, 0^{1}, 1^{1}, 0^{2}, \ldots\right)$ and $\left(1^{2}, 1^{2}, 0^{1}, 1^{1}, 0^{2}, \ldots\right)$.

By construction, the targets $V\left(\xi_{n}, \eta_{n+l}\right)$ cut across the fundamental domains $T_{0}$ and $T_{1}$ for any $n \in \mathbb{Z}^{+}$. Consequently, we may denote by $W^{i}\left(\xi_{n}, \eta_{n+l}\right), i=1,2$, the compact connected regions $V\left(\xi_{n}, \eta_{n+l}\right) \cap T_{1}$, lying below and above $\gamma_{2 \pi i}$, respectively. The next step is to show that, by considering appropriate preimages of the sets $W^{i}\left(\xi_{n}, \eta_{n+l}\right)$, we end up with a nested sequence of neighborhoods of either $\alpha_{s, l}$ if $s$ is an $A$-sequence, or, either $\alpha_{\bar{s}_{1}}$ or $\alpha_{\bar{s}_{2}}$ if $s$ is a $B$-sequence. We note that, because of Proposition 3.2, we may assume that $s \in \Sigma_{M}$ has infinitely many 1's.

The next lemma is a restatement of Corollary 3.4 in [11].

Lemma 4.3. Let $\ell \geq 0$ and $s=\left(\tau_{0}, 1, \tau_{1}, 1, \tau_{2}, 1, \ldots\right) \in \Sigma_{M}$, where $\tau_{i}, i=0,1,2, \ldots$ are blocks of symbols in $\{-M, \ldots, 0,1, \ldots M\}$ of length $n_{i}$. Let $m_{j}=j+n_{0}+n_{1}+\ldots+n_{j}, j \geq 0$.

(a) If $s$ is an $A$-sequence and $\bar{s}$ is its unique augmentation, then the sets

$$
L_{s_{0}} \circ \cdots \circ L_{s_{m_{j}}}\left(W^{\rho\left(m_{j}\right)}\left(\xi_{m_{j}}, \eta_{m_{j}+l}\right)\right)
$$

where

$$
\rho\left(m_{j}\right)= \begin{cases}2 & \text { if } \bar{s}_{m_{j}+2} \neq\left\{0^{2}, 1^{1}\right\} \\ 1 & \text { if } \bar{s}_{m_{j}+2}=\left\{0^{2}, 1^{1}\right\}\end{cases}
$$

form a nested sequence of subsets of $V\left(\zeta, \eta_{\ell}\right)$. As $m_{j} \rightarrow \infty$ these neighborhoods tend to a portion of the curve $\omega_{s}$ that contains at least $\alpha_{s, \ell}$. 
(b) If $s$ is a $B$-sequence and $\bar{s}_{1}$ and $\bar{s}_{2}$ are the two augmentations of $s$, then

$$
L_{s_{0}} \circ \cdots \circ L_{s_{m_{j}}}\left(W^{i}\left(\xi_{m_{j}}, \eta_{m_{j}+l}\right)\right), i=1,2 \text {, }
$$

form two nested sequences of subsets of $V\left(\xi_{0}, \eta_{\ell}\right)$. As $m_{j} \rightarrow \infty$ these two families of neighborhoods tend to the portions of the curves $\omega_{\bar{s}_{1}}$ and $\omega_{\bar{s}_{2}}$ that contains at least $\alpha_{\bar{s}_{1}, \ell}$ and $\alpha_{\bar{s}_{2}, \ell}$.

Proof. We first prove (a). Since $s$ is an $A$-sequence, there is a unique augmentation $\bar{s}$. Note that the superscript corresponding to $\bar{s}_{m_{j}+1}$ depends on the next symbol, $\bar{s}_{m_{j}+2}$. More precisely, this superscript is 1 if $\bar{s}_{m_{j}+2}=0^{2}$ or $1^{1}$, or 2 otherwise.

Hence, from Proposition 4.2(b), the sets given by (1) form a nested sequence in $V\left(\zeta, \eta_{\ell}\right)$. Notice that $\left|E^{\prime}\right|>1$ in $H_{\zeta}$ and the only points in $H_{\zeta}$ whose orbits tend directly to $\infty$ with $A$-itinerary $s$ are those in $\omega_{s}$ (which is a unique curve because $s$ is an $A$-sequence).

We claim that such a nested sequence of subsets contains $\alpha_{s, \ell}, l \geq 0$. The claim is straightforward for $l=0$, since $E^{n}\left(\alpha_{s}\right) \in V\left(\xi_{n}, \eta_{n}\right), n \geq 0$ (i.e., $E^{n}\left(\alpha_{s}\right) \in W^{\rho(n)}\left(\xi_{n}, \eta_{n}\right), n \geq 0$ for the suitable $n$ and $\rho(n))$. So, when considering the $k$-preimages with $k=1, \ldots n$ of the $W$-boxes, following the sequence $s$, we found inside the $E^{n-k}\left(\alpha_{s}\right)$. The process finish at $\alpha_{s}$ itself, as desired.

To see the claim with $l>0$ we notice that in $V\left(\xi_{n}, \eta_{n+l}\right)$ we have a connected segment of $w_{s}$ including $E^{n}\left(\alpha_{s}\right) \cup \ldots \cup E^{n+l}\left(\alpha_{s}\right)$. So, the preimage argument above only shows that we finish at some portion of $\alpha_{s, l}$ (remember that $\alpha_{s, l}$ is constructed by adding the union of the successives imatges of all sequences $\left.\sigma^{-i}(s), i=1 \ldots l\right)$. To finish the proof we use the same preimages argument not only to the sequence $s$ but to all sequences of the form $\sigma^{-i}(s), i=1 \ldots l$ (that is, we take preimages of $\left.E^{n}\left(\alpha_{\sigma^{-i}(s)}\right) \cup \ldots \cup E^{n+l}\left(\alpha_{\sigma^{-i}(s)}\right), i=1, \ldots l\right)$.

Now we prove statement (b). Since $s$ is a $B$-sequence, there are exactly two augmentations, $\bar{s}_{1}$ and $\bar{s}_{2}$, corresponding to $s$. Therefore, at each $j$-step in (2), we get two subsets of $V\left(\zeta, \eta_{\ell}\right)$ depending on $i$ being equal to 1 or 2 . The rest of the argument is similar to the previous case.

Remark 4.1. In Lemma 4.3(b), each step gives two subsets of $V\left(\zeta, \eta_{\ell}\right)$ depending on $i=1,2$. It is not true in general that, as $m_{j} \rightarrow \infty$, for $i=1$ (respectively $i=2$ ) we get a nested sequence of subsets that contains precisely $\alpha_{\bar{s}_{1}, \ell}$ (respectively $\left.\alpha_{\bar{s}_{2}, \ell}\right)$, or viceversa. The lemma only states

that, as $m_{j} \rightarrow \infty$, and according to the $\bar{B}$-sequences $\bar{s}_{1}$ and $\bar{s}_{2}$, we end up with two disjoint nested sequences of subsets in $V\left(\zeta, \eta_{\ell}\right)$.

In the next section we use the above results to show the existence of different types of indecomposable continua when the sequence has strings of 1-symbols that grow exponentially fast.

\subsection{Indecomposable continua for $B$-sequences}

The first step in our construction is to find special B-sequences (that is, sequences with only 0's and 1's as entries) for which the extended tails lie arbitrarily far into the left hand half plane. We say that two continuous curves parametrized by $\left(x, h_{i}(x)\right), x>x_{0}, i=1,2$ are $\epsilon$-close if $\left|h_{1}(x)-h_{2}(x)\right|<\epsilon$, for all $x$. 
We start the construction by considering $B$-sequences of the form $1_{k} t$ where $1_{k}$ denotes a string of 1 's of length $k$, and $t=\left(t_{0}, t_{1}, t_{2}, \ldots\right)$ is any $B$-sequence not ending in all 1 's. So, $1_{k}^{1} \bar{t}_{1}$ and $1_{k}^{2} \bar{t}_{2}$ denote the two admissible augmentations corresponding to $1_{k} t$. Finally $\omega_{1_{k}^{1} \bar{t}_{1}}$ and $\omega_{1_{k}^{2} \bar{t}_{2}}$ denote the two tails corresponding to the $B$-sequence $1_{k} t$.

Let $\epsilon>0$ and let $D_{2 \pi i}^{\delta}$ be the disk surrounding $z=2 \pi i$ with radius $\delta>0$. For $k$ large enough we may assume that $\omega_{1_{k}^{i} \bar{t}_{i}}, i=1,2$ are $\epsilon$-close curves in $[\zeta, \infty)$, one below and one above $\omega_{2 \pi i}$, the tail of the hair $\gamma_{2 \pi i}$. If we pull back these two curves by applying $L_{1}$ (the inverse function of $E$ taking values in $T_{1}$ ) we obtain two new curves $\varpi_{1_{k+1}^{i}} \bar{t}_{i}, i=1,2$ whose points have itinerary $1_{k+1} t$. These curves are $\epsilon$-close over the domain $[\log \zeta, \infty]$. Continuing the pull back via successive applications of $L_{1}$, we find a first positive integer $r$ such that the two curves $\varpi_{1_{k+r}^{i} \bar{t}_{i}}, i=1,2$ are $\epsilon$-close to each other (and to $\gamma_{2 \pi i}$ ), extend to $\infty$ to the right, and their left hand end lie inside $D_{2 \pi i}^{\delta}$.

At this stage, we pull back the curves $\varpi_{1_{k+r}^{i}} \bar{t}_{i}$ once more but now using $L_{0}$ (the inverse function of $E$ taking values in $T_{0}$ ). We get two new curves, denoted by $\varpi_{0^{i} 1_{k+r}^{i}} \bar{t}_{i}, i=1,2$, one below and one above $\gamma_{0}$, respectively. These curves extend to $\infty$ to the right and their left hand end lie inside $D_{0}^{\delta}$ (that is, the disk centered at $z=0$ of radius $\delta$ ). The points in $\varpi_{0^{i} 1_{k+r}^{i}} \bar{t}_{i}$ follow the $\bar{B}$-sequence $0^{i} 1_{k+r}^{i} \bar{t}_{i}, i=1,2$.

Finally, we pull back the two curves once again by using $L_{0}$ to obtain two curves in $T_{0}$. The two curves, $\varpi_{0^{1} 0^{2} 1_{k+r}^{2} \bar{t}_{2}}$ and $\varpi_{0^{2} 0^{1} 1_{k+r}^{1} \bar{t}_{1}}$, which we call extended tails, contain the two tails $\omega_{0^{1} 0^{2} 1_{k+r}^{2} \bar{t}_{2}}$ and $\omega_{0}^{2} 0^{1} 1_{k+r}^{1} \bar{t}_{1}$, and points on them follow the $B$-sequence $001_{k+r} t$ (precisely, in terms of $\bar{B}_{-}$ sequences, they follow $0^{1} 0^{2} 1_{k+r}^{2} \bar{t}_{2}$ and $0^{2} 0^{1} 1_{k+r}^{1} \bar{t}_{1}$, respectively). They extend arbitrarily far to the left as long as we start all the construction with a $k$ large enough. Consequently they are part of $\gamma_{0^{1} 0^{2} 1_{k+r}^{2} \bar{t}_{2}}$ and $\gamma_{0^{2} 0^{1}} 1_{k+r}^{1} \bar{t}_{1}$, respectively. In short, we have shown that

Lemma 4.4. Given any $\mu<0$, there exits $K>0$ such that for all $k>K$, there exist two continuous curves, $\varpi_{0^{1} 0^{2} 1_{k}^{2} \bar{t}_{2}}$ and $\varpi_{0^{2} 0^{1} 1_{k}^{1} \bar{t}_{1}}$, satisfying:

(a) these curves are the extended tails that follow the $\bar{B}$-sequences $0^{1} 0^{2} 1_{k}^{2} \bar{t}_{2}$ and $0^{2} 0^{1} 1_{k}^{1} \bar{t}_{1}$, respectively; and

(b) they cross $\operatorname{Re} z=\mu$ in the far left half plane.

We wish to show that, for any $n$, the forward image of the targets $V\left(\xi_{n}, \eta_{n}\right)$ map in a convenient way onto the two curves constructed above. We say that $\vartheta_{k}=\left(\theta_{0}, \theta_{2}, \ldots, \theta_{k-1}\right)$ is a $B$-block of length $k$ if it is an string of $B$-symbols of length $k$. Let $\bar{\vartheta}_{k, 1}$ and $\bar{\vartheta}_{k, 2}$ denote the two $\bar{B}$-blocks of length $k$, correspondingly.

Proposition 4.5. Let $l \geq 0, k>0$ and $n>0$. Let $t$ be any $B$-sequence and $\vartheta_{n}$ be any $B$-block of length $n$. Let $\Theta=\vartheta_{n} 1001_{k}$ t be a $B$-sequence and let $\bar{\Theta}_{1}=\bar{\vartheta}_{n, 1} 1^{1} 0^{2} 0^{1} 1_{k}^{1} \bar{t}_{1}$ and $\bar{\Theta}_{2}=\bar{\vartheta}_{n, 2} 1^{2} 0^{1} 0^{2} 1_{k}^{2} \bar{t}_{2}$ its augmentations. Then there exist $K>0$ such that for all $k>K$, the following statements hold. 
(a) The forward image of $W^{2}\left(\xi_{n}, \eta_{n+l}\right)$ cuts twice (once far to the left and once far to the right) across the extended tail $\varpi_{0^{1} 0^{2} 1_{k}^{2} \bar{t}_{2}}$, and cuts once (to the left) across the extended tail $\varpi_{0^{2} 0^{1} 1_{k}^{1} \bar{t}_{1}}$.

(b) The forward image of $W^{1}\left(\xi_{n}, \eta_{n+l}\right)$ cuts once (to the right) across the extended tail $\varpi_{0^{2} 0^{1} 1_{k}^{1} \bar{t}_{1}}$.

(c) The hair $\gamma_{\bar{\Theta}_{2}}$ passes twice and the hair $\gamma_{\bar{\Theta}_{1}}$ passes once through

$$
L_{\theta_{0}} \circ \ldots \circ L_{\theta_{n-1}}\left(W^{2}\left(\xi_{n}, \eta_{n+l}\right)\right) \text {. }
$$

(d) The hair $\gamma_{\bar{\Theta}_{1}}$ passes once through

$$
L_{\theta_{0}} \circ \ldots \circ L_{\theta_{n-1}}\left(W^{1}\left(\xi_{n}, \eta_{n+l}\right)\right)
$$

Remark 4.2. The piece of the hair $\gamma_{\bar{\Theta}_{1}}$ passing through the set defined in (3) follows a "non admissible" $\bar{B}$-sequence, precisely, $\bar{\vartheta}_{n, 1} 1^{2} 0^{1} 0^{2} 1_{k}^{2} \bar{t}_{2}$ (from Remark 3.1 we see that $1^{2}$ cannot be followed by $\left.0^{1}\right)$. Although this seems a contradiction, we notice that these points follow such itinerary but they do not tend directly to infinity. So, the admissibility condition (introduced in Remark 3.1) does not apply. Formally, after $n+1$ iterates of the exponential this piece of the hair is mapped to the left hand side of the extended tail $\varpi_{0^{1} 0^{2} 1_{k}^{2} \bar{t}_{2}}$. Hence they follow the desired $B$-itinerary (even though the $\bar{B}$-itinerary is not admissible), by moving from right to left in $\mathbb{C}$.

Proof. Consider the region $T_{0} \cap H_{\zeta}$. We denote by $U_{0^{1}}$ and $U_{0^{2}}$ the two subregions in $T_{0} \cap H_{\zeta}$ that lie below and above of the tail of $\gamma_{0}$ respectively. Similarly, define the regions $U_{1^{1}}$ and $U_{1^{2}}$ inside the set $T_{1} \cap H_{\zeta}$. Let us fix $n$.

Clearly, $E\left(W^{2}\left(\xi_{n}, \eta_{n+l}\right)\right)$ is a portion of an annulus originating at $\gamma_{2 \pi i}$ and ending at $\gamma_{0}$ from below. Hence, this image crosses $T_{0}$ far to the left and crosses $U_{0^{1}}$ far to the right. On the other hand, $E\left(W^{1}\left(\xi_{n}, \eta_{n+l}\right)\right)$ consists of a portion of a "rectangle" far to the right, extending from $\gamma_{0}$ to $\gamma_{2 \pi i}$ from below (that is, the image cuts across $U_{0^{2}} \cap U_{1^{1}}$ ). See Figure 3.

Therefore, statements (a) and (b) are easy corollaries of Lemma 4.4, since the extended tails $\varpi_{0^{1} 0^{2} 1_{k}^{2} \bar{t}_{2}}$ and $\varpi_{0^{2} 0^{1} 1_{k}^{1} \bar{t}_{1}}$ extend arbitrarily far to the left as long as $k$ is large enough.

We claim now that statements (c) and (d) follow from the above statements (a) and (b), and Lemma 4.3(b). To see this we note that for the suitable $k$ defined above, the following statements hold.

(i) There are in $W^{2}\left(\xi_{n}, \eta_{n+l}\right)$ two different pieces of the hair $\gamma_{\Theta_{2}}$. One is mapped by $E$ to the right hand side of the extended tail $\varpi_{0^{1} 0^{2} 1_{k}^{2} \bar{t}_{2}}$ (that is, the tail of the hair $\gamma_{0^{1} 0^{2} 1_{k}^{2} \bar{t}_{2}}$ ), and the other is mapped by $E$ to the left hand side of the extended tail $\varpi_{0^{1} 0^{2} 1_{k}^{2} \bar{t}_{2}}$.

(ii) There is in $W^{2}\left(\xi_{n}, \eta_{n+l}\right)$ a piece of the hair $\gamma_{\Theta_{1}}$, mapped by $E$ to the left hand side of the extended tail $\varpi_{0^{2} 0^{2} 1_{k}^{2} \bar{t}_{2}}$. Note this piece follows the non admissible $\bar{B}$-sequence $1^{2} 0^{2} 0^{1} 1_{k}^{1} \bar{t}_{1}$ as it was explained in Remark 4.2. 


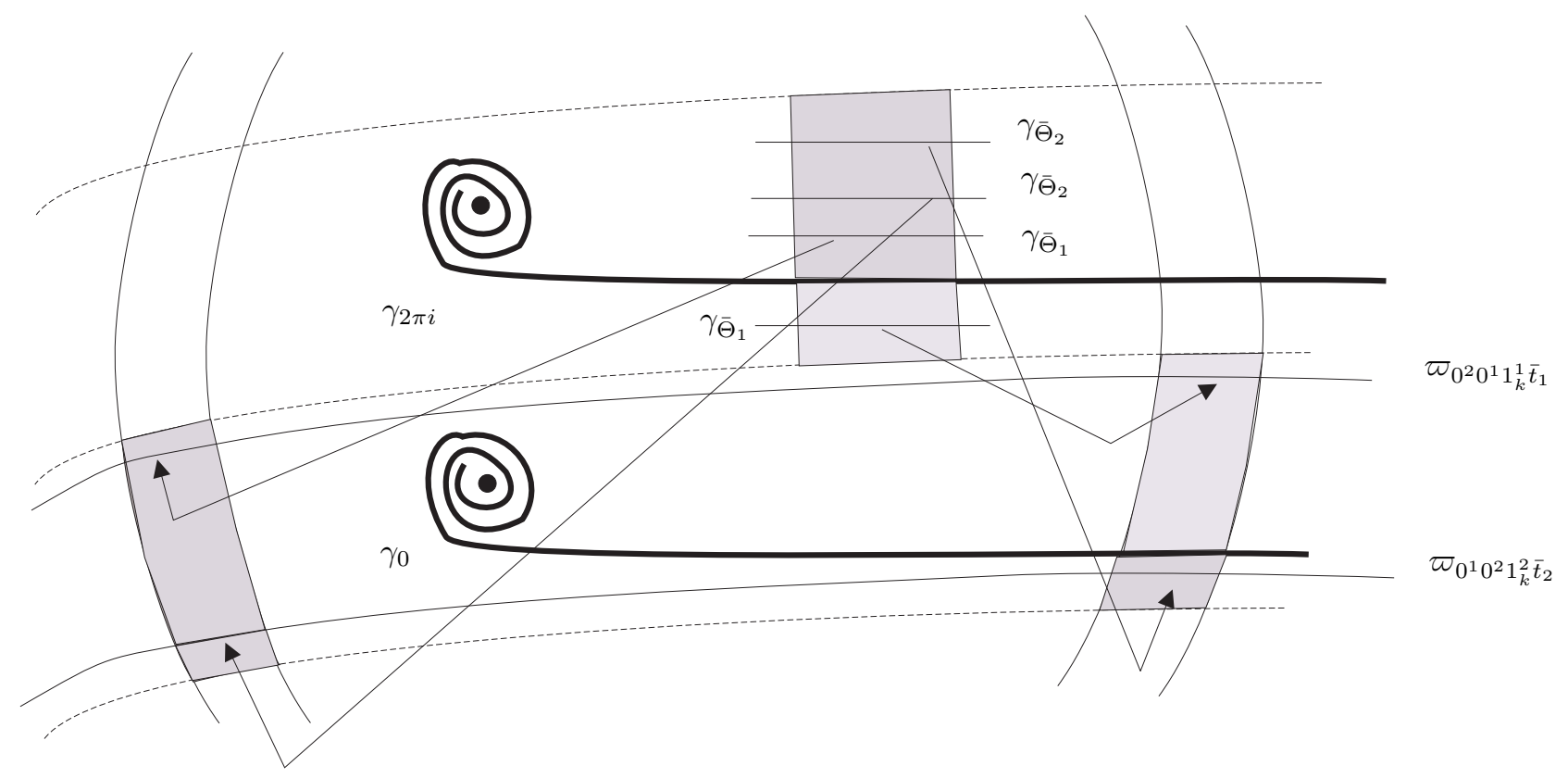

Figure 3: The geometric explanation of Proposition 4.5. The arrows denote the forward image of the pieces of $\gamma_{\overline{\theta_{1}}}$ and $\gamma_{\overline{\theta_{2}}}$.

(iii) There is in $W^{1}\left(\xi_{n}, \eta_{n+l}\right)$ one piece of the hair $\gamma_{\Theta_{1}}$, which is mapped by $E$ to the right hand side of the extended tail $\varpi_{0^{2} 0^{1} 1_{k}^{1} \bar{t}_{1}}$ (that is, the tail of the hair $\gamma_{0^{2} 0^{1} 1_{k}^{1} \bar{t}_{1}}$ ). Since the extended tail $\varpi_{0^{2} 0^{1} 1_{k}^{1} \bar{t}_{1}}$ is a connected set and $E$ is a continuous map, the two pieces of the hair $\gamma_{\Theta_{1}}$ lying in $W^{1}\left(\xi_{n}, \eta_{n+l}\right)$ and $W^{2}\left(\xi_{n}, \eta_{n+l}\right)$ are connected to each other.

(iv) Pulling back $W^{i}\left(\xi_{n}, \eta_{n+l}\right), i=1,2$ by following the $B$-block $\vartheta_{n}$ as in Lemma 4.3 , we produce the desired nested subsets of $V\left(\eta_{0}, \mu_{l}\right)$.

Remark 4.3. Note that all of the previous results hold for infinitely many B-sequences, since we use an arbitrary $B$-sequence $t$ that does not terminate in all 1's.

Now we state and prove the main result of this paper.

Theorem 4.6. Given an infinite sequence of $B$-blocks $\left\{\tau_{m_{1}}, \tau_{m_{2}}, \ldots\right\}$ we may find an increasing sequence of integers $k_{j}$ such that the $B$-sequence s given by

$$
s=\tau_{m_{1}} 1001_{k_{1}} \tau_{m_{2}} 1001_{k_{2}} \tau_{m_{3}} \ldots
$$

is admissible and has the property that $I(s)$ consists of either an indecomposable continuum in the Riemann sphere and a distinguished curve that accumulates on it, or else the closure of a pair of 
curves that accumulate on themselves as well as on each other. In this case the set of accumulation points is an indecomposable continuum. The fact that we have one case or the other only depends on the initial sequence of $B$-blocks $\left\{\tau_{m_{1}}, \tau_{m_{2}}, \ldots\right\}$.

Proof. Let $l \geq 0, k_{0}=0$ and define

$$
p_{i}=m_{1}+\ldots+m_{i}+k_{1}+\ldots+k_{i-1}+3(i-1)+1, \quad i>0 .
$$

We also construct inductively $B$-sequences $\beta_{j}, j>0$, given by

$$
\beta_{j}=\tau_{m_{1}} 1001_{k_{1}} \tau_{m_{2}} 1001_{k_{2}} \ldots \tau_{m_{j}} 1001_{k_{j}} t,
$$

in the following way. First, let $t$ be any $B$-sequence not ending in $\overline{1}$, and note that each $p_{i}$ term in $\beta_{j}, i \leq j$, corresponds to the symbol 1 . From Proposition 4.5, we choose $k_{j}$ (at each step) in such a way that the forward image of $W^{2}\left(\xi_{p_{j}}, \eta_{p_{j}+l}\right)$ cuts across all extended tails $\varpi_{0^{1} 0^{2} 1_{k_{j}}^{2} \bar{t}_{2}}$ twice (one far to the left and one far to the right), and cuts across the extended tails $\varpi_{0^{2} 0^{1}} 1_{k_{j}} \bar{t}_{1}$ once far to the left. Moreover, $W^{1}\left(\xi_{p_{j}}, \eta_{p_{j}+l}\right)$ cuts across the extended tails $\varpi_{0^{2} 0^{1} 1_{k_{j}} \bar{t}_{1}}$ only once.

As $j$ tends to infinity, the limit of this inductive construction defines a $B$-sequence $s$ for which $I(s)$ will satisfy the statements of the theorem. Nevertheless, the above construction does not define $s$ uniquely, because the blocks $1_{k_{j}}$ can be chosen arbitrarily as stated in Proposition 4.5, but we fix one of those possible choices.

Let $\overline{s_{1}}$ and $\overline{s_{2}}$ be the augmentations of $s$ and similarly, let $\bar{\beta}_{j, 1}, \bar{\beta}_{j, 2}$ be the augmentations of $\beta_{j}$. By construction the $p_{j}$ terms in each $\bar{s}_{i}$ (similarly in each $\bar{\beta}_{j, i}$ ) are either $1^{1}$ or $1^{2}$. Moreover, if it is $1^{1}$ for $\bar{s}_{1}$ (respectively $\bar{\beta}_{j, 1}$ ), then it is $1^{2}$ for $\bar{s}_{2}$ (respectively $\bar{\beta}_{j, 2}$ ), and viceversa.

To see this we divide the proof into two cases.

Case (i). Each $\bar{s}_{k}$ may have a finite number (possibly zero) of symbols $1^{1}$ or $1^{2}$ at the $p_{j}$ th position. We may assume, without loss of generality, that all of the $p_{j}$ terms in $\bar{s}_{1}$ are $1^{2}$ symbols, and consequently all of the $p_{j}$ terms in $\bar{s}_{2}$ are $1^{1}$ symbols.

Let $j>0$. By construction, we must have three disjoint pieces of curves in $W^{2}\left(\xi_{p_{j}}, \eta_{p_{j}+l}\right)$ satisfying the following: Two of them land after one iteration on the extended tail following the $\bar{B}$-sequence $\sigma^{p_{j}+1}\left(\bar{s}_{1}\right)$; one far to the left and one far to the right (remember that, by hypothesis, $\bar{s}_{1}$ is such that has a $0^{1}$ in the $p_{j}+1$ term). Of course, these two pieces belong to the same extended tail since they are disjoint parts of the preimage of an extended (connected) tail. The third piece lands (after one iteration) on the left hand side of the extended tail following the nonadmissible $\bar{B}$-sequence, $\sigma^{p_{j}+1}\left(\bar{s}_{2}\right)$. Moreover we must have in $W^{1}\left(\xi_{p_{j}}, \eta_{p_{j}+l}\right)$ a unique piece of the curve landing (after one iteration) on the extended tail following the $\bar{B}$-sequence $\sigma^{p_{j}+1}\left(\bar{s}_{2}\right)$.

So, if we regard the $B$-block $\tau_{m_{1}} 1001_{k_{1}} \tau_{m_{2}} \ldots t_{m_{j}}$ as the $B$-block $\vartheta_{n}$ in Proposition 4.5 , and Lemma 4.3(b) is applied, it is easy to see that, after going back through the suitable inverses of the exponential (following the $B$-itinerary), we end up in one of the two subsets of $V\left(\eta_{0}, \mu_{l}\right)$ corresponding to the preimages of $W^{i}\left(\xi_{p_{j}}, \eta_{p_{j}+l}\right), i=1,2$. One of them (the preimage of $W^{2}\left(\xi_{p_{j}}, \eta_{p_{j}+l}\right)$ ) includes $\alpha_{s_{l, 1}}$ as well as two further pieces of curves having the desired $B$ itinerary. Indeed, one 
of them is a piece of the hair $\gamma_{\bar{s}_{1}}$ and the other is a piece of the hair $\gamma_{\bar{s}_{2}}$. The other subset (the preimage of $\left.W^{1}\left(\xi_{p_{j}}, \eta_{p_{j}+l}\right)\right)$ includes $\alpha_{s_{l, 2}}$.

Since we are assuming that $\bar{s}_{1}$ has $1^{2}$ in all of the $p_{j}$ terms, as $j \rightarrow \infty$, the above construction gives two nested sequences of subsets of $V\left(\eta_{0}, \mu_{l}\right)$ satisfying the same statement at each step, in the sense that one of the nested sequence includes $\alpha_{s_{l, 1}}$ (a piece of the hair $\gamma_{\overline{s_{1}}}$ ) as well as another piece of the hair $\gamma_{\overline{s_{1}}}$, and a piece of the hair $\gamma_{\bar{s}_{2}}$. The other nested sequence includes $\alpha_{s_{l, 2}}$. In summary, we have shown that the hairs $\gamma_{\overline{s_{1}}}$ and $\gamma_{\overline{s_{2}}}$ accumulate on the tail $\gamma_{s_{1}}$ (since they accumulate on $\left.\bar{s}_{l, 1}, l \geq 0\right)$ while the hair $\gamma_{\overline{s_{2}}}$ does not accumulate onto itself.

To see that $\gamma_{\bar{s}_{1}}$ and $\gamma_{\bar{s}_{2}}$ indeed accumulate on points in $\gamma_{\bar{s}_{1}}$ that do not lie in the tail, we note that we may perform the same construction for the sequences

$$
\tau_{m_{i}} 1001_{k_{i}} \tau_{m_{i+1}} 1001_{k_{i+1}} \tau_{m_{i+2}} \ldots, i=1,2,3, \ldots
$$

Then we may pull the corresponding hairs and their accumulations back by the appropriate logarithms to show that $\gamma_{\bar{s}_{1}}$ as well as $\gamma_{\bar{s}_{2}}$ must accumulate on any point in the hair $\gamma_{\bar{s}_{1}}$. Consequently, $I(s)$ is the union of a curve that accumulates on itself and another curve that accumulates on the first curve.

Case (ii). Here we may assume the symbols $1^{1}$ and $1^{2}$ appear infinitely many times in the $p_{j}$ th positions for both augmented sequences $\bar{s}_{1}$ and $\bar{s}_{2}$. Consequently, we may consider two infinite subsequences, denoted by $p_{j_{1}}$ and $p_{j_{2}}$, such that the terms $p_{j_{1}}$ of $\bar{s}_{1}$ are all $1^{2}$ while the terms $p_{j_{2}}$ of $\bar{s}_{2}$ are all $1^{2}$.

We may now apply a similar argument to the $p_{j_{k}}$ subsequences. So, if we regard the $B$-block $\tau_{m_{1}} 1001_{k_{1}} \tau_{m_{2}} \ldots t_{m_{j_{k}}}$ as the $B$-block $\vartheta_{n}$ in Proposition 4.5, and apply Lemma 4.3(b), it is easy to see that after pulling back through the suitable inverses of the exponential, we end up in two subsets of $V\left(\eta_{0}, \mu_{l}\right)$, namely, the corresponding preimages of $W^{i}\left(\xi_{p_{j_{k}}}, \eta_{p_{j_{k}}+l}\right), i=1,2$. The preimage of $W^{2}\left(\xi_{p_{j_{k}}}, \eta_{p_{j_{k}}+l}\right)$ will include $\alpha_{s_{l, k}}$ (a piece of the hair $\gamma_{s_{k}}$ ) as well as pieces of the hairs $\bar{s}_{1}$ and $\bar{s}_{2}$. On the other hand, the preimage of $W^{1}\left(\xi_{p_{j_{k}}}, \eta_{p_{j_{k}}+l}\right)$ will include $\alpha_{s_{l, d}}, d \neq k$ (a piece of the hair $\left.\gamma_{s_{d}}\right)$.

It follows that each of the hairs $\overline{s_{1}}$ and $\overline{s_{2}}$ accumulates on itself as well as each other. Hence the set of accumulation points of both curves is an indecomposable continuum.

Finally we claim that these hairs do not separate the plane. If this were not the case, then one of the complementary domains would necessarily be a wandering domain. This contradicts Sullivan's Theorem. See [17], [23].

Remark 4.4. The case of $A$-sequences can also be studied to find indecomposable continua under certain (similar) conditions on the s sequence. However it does not introduce any new phenomena (in the topology of the indecomposables). 


\section{Dynamics for $B$-sequences}

Previous work related to the topology and dynamics of the set $I(s)$ that appear in [7], [11] and [21] have shown the duality within the complexity of the topology and the simplicity of the dynamics. Our case is no exception to this rule although the dynamics for $\lambda=2 \pi i$ has a peculiar behavior not found in previous cases. Indeed, there is no point with bounded orbit following a sequence like in Theorem 4.6 .

Lemma 5.1. Let $s=\tau_{m_{1}} 1001_{k_{1}} \tau_{m_{2}} 1001_{k_{2}} \tau_{m_{3}} \ldots$ as in Theorem 4.6. Then, any point $z \in I(s)$ has an unbounded orbit.

Proof. The result is trivial if $z$ is a point in one of the hairs $\gamma_{s_{j}}, j=1,2$. So assume $z \in I(s)-\left(\gamma_{s_{1}} \cup\right.$ $\gamma_{s_{2}}$ ) and we proceed by contradiction. Suppose there exist real numbers $-\infty<a<0<b<\infty$, so for $i=0,1$, we can define the regions

$$
Q_{i}=\{z \mid a \leq \operatorname{Re} z \leq b\} \cap T_{i}
$$

whose union contain the orbit of $z$. Note that the left hand boundary of these regions, $\operatorname{Re}(z)=a$, is mapped to a circle around 0 of radius $r=|E(a)|$. Denote by $D(0, r)$ the open disk around $z=0$ of radius $r$ and assume $D(0, r)$ is completely contained in $R_{0}$ (otherwise we may adjust the value of $a$ to accomplish this). Note that the region $\operatorname{Re}(z)<a$ is mapped to the interior of this disk. Let $D(2 \pi i)$ denote the image of $D(0, r)$ under $E$ and again, assume $D(2 \pi i)$ is completely contained inside $Q_{1}$.

Let $L_{1}$ denote the branch of $E^{-1}$ restricted to the strip $T_{1}$. Note $L_{1}$ is an strict contraction inside $Q_{1}$. Then, since $2 \pi i$ is the only repelling fixed point in $Q_{1}$, we may find an integer $N>0$ large enough so

$$
L_{1}^{N}\left(Q_{1}\right) \subset D(2 \pi i) .
$$

Since the sequence $k_{n}$ in increasing and unbounded, we can find an integer $n_{0}$ for which $k_{n}>N$ for each $n>n_{0}$. Let $z_{j}=E^{j}(z)$ be the point in the orbit of $z$ that follows the itinerary $1_{k_{n}} \tau_{m_{n+1}} 100 \ldots$ This implies that $z_{j} \in D(2 \pi i)$ and thus, the point $z_{j-2}$ that follows the itinerary $001_{k_{n}} \tau_{m_{n+1}} 100 \ldots$ must lie to the left of the region $Q_{0}$, giving us a contradiction.

Now we state the main result of this section.

Theorem 5.2. Assume $s$ is a sequence that satisfies the conditions of Theorem 4.6. Let $\overline{s_{1}}$ and $\overline{s_{2}}$ be the two possible augmentations of $s$ and let $z \in I(s)$. Then,

(a) If $z$ is a point in one of the hairs $\gamma_{\overline{s_{1}}}$ or $\gamma_{\overline{s_{2}}}$, then the $\omega$-limit set of its orbit is the point at infinity.

(b) Otherwise, the $\omega$-limit set of the orbit of $z$ contains the orbit of 0 and the point at infinity. 
Proof. Assume $z$ belongs to one of the hairs $\gamma_{\overline{s_{j}}}$ and let $\omega_{\overline{s_{j}}}$ denote the tail associated to this hair. By definition, every point in $\omega_{\overline{s_{j}}}$ escapes to infinity. Moreover, $\gamma_{\overline{s_{j}}}$ is constructed by successive pullbacks of its tail. Hence, every point in the hairs are wandering points with the point at infinity as its $\omega$-limit set. So, statement (a) is proved.

Statement (b) is a consequence of Lemma 5.1. If $z$ does not belong to any of the hairs $\gamma_{\overline{s_{j}}}, j=$ 1,2 , then it cannot escape directly to infinity inside $H_{\zeta}$. However, since it follows the itinerary given by the sequence $s$, from Lemma 5.1, its orbit cannot be bounded. So it must visit points with arbitrary big negative real part (inside $T_{0} \cup T_{1}$ ). Consequently it must pass through any arbitrarily small neighborhood of 0 . Of course this also implies that the orbit passes arbitrarily close to $z=2 \pi i$ and infinity, as desired.

\section{References}

[1] Aarts, J. M. and Overteegen, L. G. The Geometry of Julia Sets. Trans. Amer. Math. Soc., 338, no. 2, (1993) 897-918.

[2] Barge, M., Horseshoe Maps and Inverse Limits. Pacific Journal of Mathematics. 121 (1986), 29-39.

[3] Bhattacharjee, R. and Devaney, R. L. Tying Hairs for Structurally Stable Exponentials. Ergodic Theory and Dynamical Systems 20 (2000), 1603-1617.

[4] Blanchard, P. Complex Analytic Dynamics on the Riemann Sphere. Bulletin of the American mathematical Society. 11 No.1, (1984), 85-141.

[5] Bodelón, C., Devaney, R. L., Goldberg, L., Hayes, M., Hubbard, J., and Roberts, G. Hairs for the Complex Exponential Family. Intl. J. Bifurcation and Chaos 9 (1999), 1517-1534.

[6] Curry, S. One-dimensional Nonseparating Plane Continua with Disjoint $\epsilon$-dense Subcontinua. Topol. and its Appl. 39 (1991), 145-151.

[7] Devaney, R. L. Knaster-like Continua and Complex Dynamics. Ergodic Theory and Dynamical Systems 13 (1993), 627-634.

[8] Devaney, R. L. Se $e^{x}$ Dynamics, Topology, and Bifurcation of Complex Exponentials. Topology Appl. 110 (2001), 133-161.

[9] Devaney, R. L. and Durkin, M. The Exploding Exponential and Other Chaotic Bursts in Complex Dynamics. Amer. Math. Monthly 98 (1991), 217-233. 
[10] Devaney, R. L. and Jarque, X. Misiurewicz Points for Complex Exponentials Int. J. Bifurcation and Chaos 7 (1997), 1599-1616.

[11] Devaney, R. L. and Jarque, X. Indecomposable Continua in Complex Dynamics. Conform. Geom. Dyn. (2002) 1-12 (electronic).

[12] Devaney, R. L. and Krych, M. Dynamics of Exp(z), Ergodic Theory and Dynamical Systems 4 (1984), 35-52.

[13] Deville, R. E. L., Itineraries of Entire Functions. To appear in J. Difference Eq.

[14] Eremenko, A. and Lyubich, M. Yu. Dynamical Properties of Some Classes of Entire Functions. Ann. Inst. Fourier (Grenoble) 42 no. 4, (1992), 989-1020.

[15] Fatou, P. Sur l'Itération des Fonctions Transcendentes Entières, Acta Math. 47 (1926), 337-370.

[16] Fagella, N. Limiting Dynamics for the Complex Standard Family. Inter. J. Bifur. Chaos 5 (1995), 673-699.

[17] Goldberg, L. and Keen, L. A finitness theorem for a dynamical class of entire functions. Ergodic theory and dynamical systems. 6, 183-192.

[18] Mayer, J. An Explosion Point for the Set of Endpoints of the Julia Set of $\lambda \exp (z)$, Ergodic Theory and Dynamical Systems 10 (1990), 177-184.

[19] McMullen, C. Area and Hausdorff Dimension of Julia Sets of Entire Functions. Trans. A.M.S. 300 (1987), 329-342.

[20] Misiurewicz, M. On Iterates of $e^{z}$, Ergodic Theory and Dynam. Syst. 1 (1981), 103-106.

[21] Moreno Rocha, M. Existence of Indecomposable Continua for Unstable Exponentials. Topology Proceedings, 27, (2002).

[22] Schliecher, D., Personal communication.

[23] Sullivan, D., Quasiconformal homeomorphisms and dynamics. Annals of Mathematics. 122, 401-418. 


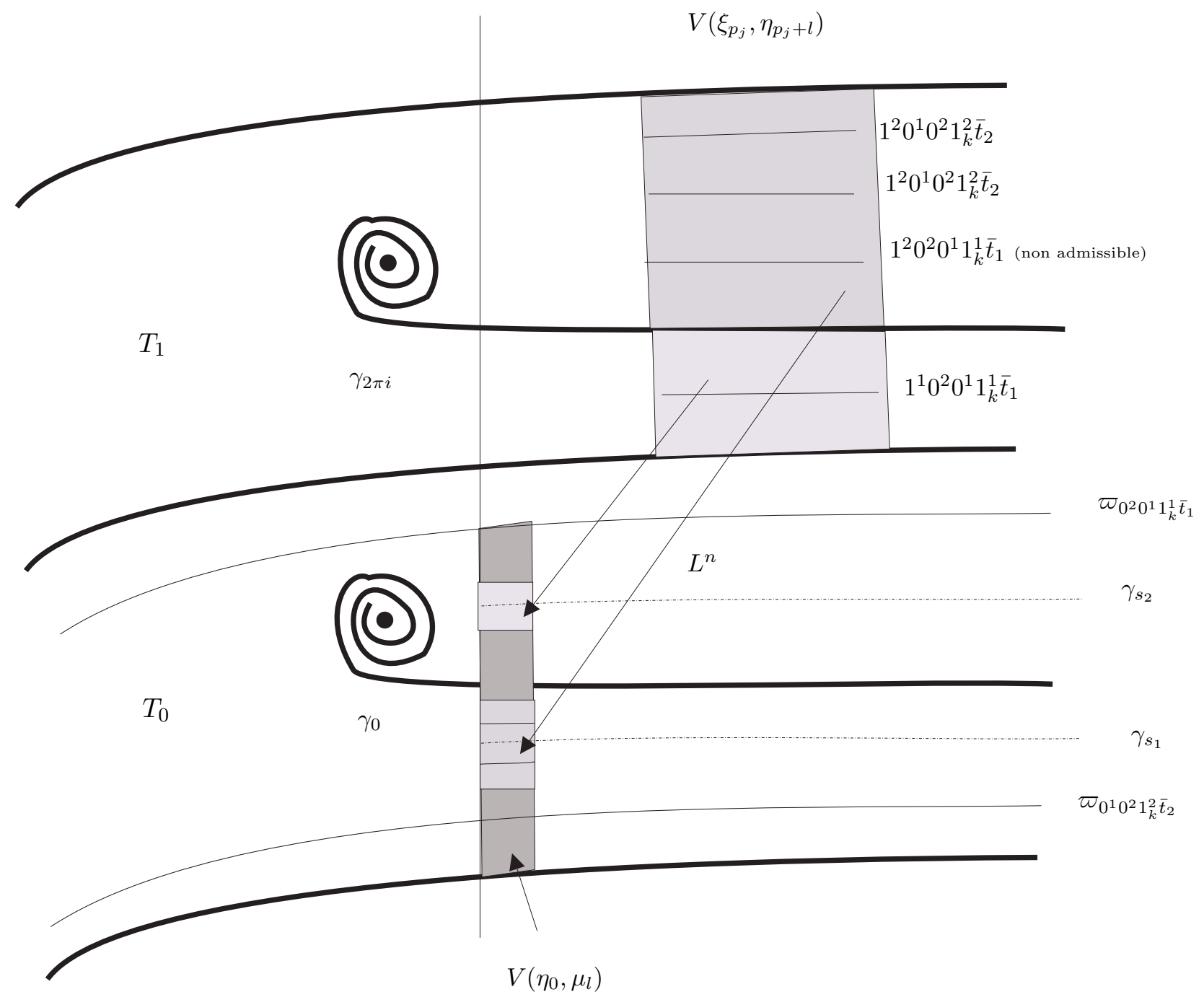

Figure 4: The geometric explanation of Theorem 4.6. We assume that the $B$-sequence starts with the symbol 0 . After taking the preimages of $V\left(\xi_{p_{j}}, \eta_{p_{j}+l}\right)$ by $E$, we find a nested sequence of neighborhoods of the two bases of the tails $\alpha_{s_{1, l}}$ and $\alpha_{s_{2, l}}$ in $V\left(\eta_{0}, \mu_{l}\right)$ 